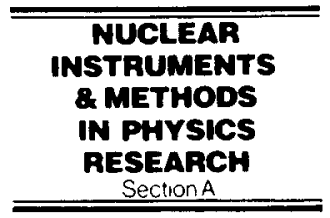

\title{
Beam test of a hadron calorimeter made of high-pressure gas-ionization tubes
}

\author{
S.L. Bagdasarov ${ }^{\text {a }}$, P.T. Cox ${ }^{\text {a }}$, L. Demortier ${ }^{\text {a }}$, N.D. Giokaris ${ }^{\text {a }}$, D.M. Khazins ${ }^{\mathrm{a}, *}$, \\ M.G. Albrow ${ }^{\text {b }}$, D.F. Anderson ${ }^{\text {b }}$, S. Cihangir ${ }^{\text {b }}$, J. Zimmerman ${ }^{\text {b }}$, G. Fanourakis ${ }^{\text {c,1 }}$, \\ F. Lobkowicz ${ }^{c}$, H.R. Gustafson ${ }^{\text {d }}$, J.A. Budagov ${ }^{\text {e }}$, S. Tokar ${ }^{e, 2}$, M. Morgan ${ }^{\text {f }}$ \\ ' Experimental Physics Department, The Rockefeller Untversity, New York, NY 10021-6399, USA \\ ${ }^{\mathrm{b}}$ Fermi National Accelerator Laboratory, Batavia, IL 60510, USA \\ ${ }^{c}$ Department of Physics and Astronomy, Unicersity of Rochester, Rochester, NY 14627-0171, USA \\ ${ }^{d}$ Unwersity of Michigan, Ann Arbor, MI 48109-1120, USA \\ e Joint Institute for Nuclear Research, Dubna, PO Box 79, 101000 Moscow, Russian Federation \\ ${ }^{\mathrm{f}}$ Ability Engineering Technology, Inc., South Holland, IL 60473, USA
}

Received 13 June 1994

\begin{abstract}
A hadron calorimeter based on high-pressure gas-ionization tubes arranged nearly parallel to the incident particle direction was built and tested in pion and electron beams at the CERN SPS. Data were taken in the energy range of 10 to $100 \mathrm{GeV}$ with the calorimeter tilted to the beam axis at angles varying from $0.9^{\circ}$ to $9.1^{\circ}$. Results of the calorimeter response to pions and electrons and its energy resolution as a function of electric field, gas pressure, beam energy, and tilt angle are presented. The calorimeter was developed for the forward region of high energy and high luminosity hadron colliders. It proved to be simple and easy to operate. Its parameters meet the needs of forward calorimetry for LHC collider experiments.
\end{abstract}

\section{Introduction}

The motivation for considering high-pressure noble-gas ionization calorimeters has been discussed in our previous papers [1-6]. (For a complete list of references on highpressure calorimeters see Ref. [6].) These calorimeters are radiation hard because they have no gas amplification and are made of radiation hard materials. Our recent measurements [7] demonstrate the ability of such a detector to resist a radiation dose as high as $1 \mathrm{Grad}$. The high electron mobility in commonly used gas mixtures yields an output signal comparable in width to that of a plastic scintillator counter. The lack of gas amplification is also responsible for the excellent linearity of these detectors. The small signal from conventional gas-ionization chambers is com-

\footnotetext{
* Corresponding author. Permanent address: Fermilab, MS 233, PO Box 500, Batavia, IL 605010, USA.

${ }^{1}$ Present address: University of Crete, Physics Department, 71409 Iraclion Crete, Greece.

${ }^{2}$ Present address: Faculty of Mathematics and Physics, Comenius University, Bratislava, 84215 , Slovakia.
}

pensated in our case by the high pressure of the working gas and by the high energy of the incident particles in the forward region. At the same time the increased gas density dramatically suppresses the effect of the so-called "Texas Towers" (large signals caused by slow neutrons in one atmosphere gas calorimeters [8]).

Several parallel-plate electromagnetic, EM [2,3,9], and hadron [10] calorimeters have been tested so far. In this paper we describe the construction and beam-test results of the first hadron calorimeter to be built of high-pressure gas-ionization tubes arranged nearly parallel to the incident particle direction. This design was motivated by both safety and signal collection considerations. The features and basic characteristics of the design are discussed elsewhere [6]. The calorimeter was built as a prototype for the high radiation environment of the forward region of highenergy hadron colliders. Independently, an EM prototype tube calorimeter has been constructed and tested by a Serpukhov group [11].

\section{Calorimeter construction}

The design of a single tube ionization chamber is shown in Fig. 1. It consists of a $12.7 \mathrm{~mm}$ diameter, $3 \mathrm{~m}$ 

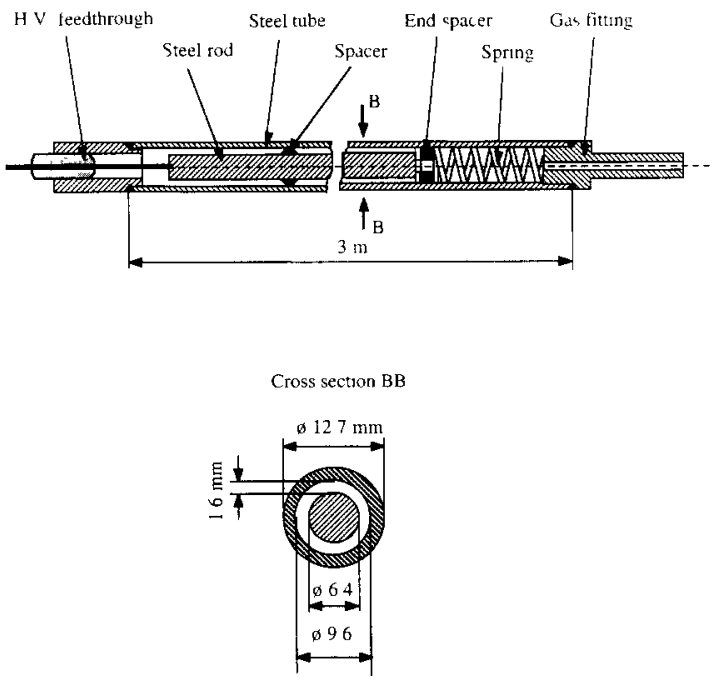

Fig. 1. Schematic of a single tube ionization chamber.

long carbon steel tube with a $9.6 \mathrm{~mm}$ diameter hole. A 6.4 mm diameter steel rod at the center of the tube is held at a positive potential to collect the electrons produced by ionization. Insulating ring spacers center the rod approximately every $50 \mathrm{~cm}$ and ensure a gap of $1.6 \mathrm{~mm}$. The spacers have narrow slots to allow gas passage. A highvoltage, high-pressure feedthrough is welded to one end of the tube. The opposite end of the tube has a gas fitting and a spring which ensures electrical contact between the rod and the feedthrough pin. The tube is welded to an adapter gas fitting. A calorimeter module (Fig. 2) is made by connecting 64 tubes to a gas manifold at the front end (facing incoming particles). The gas fittings of peripheral

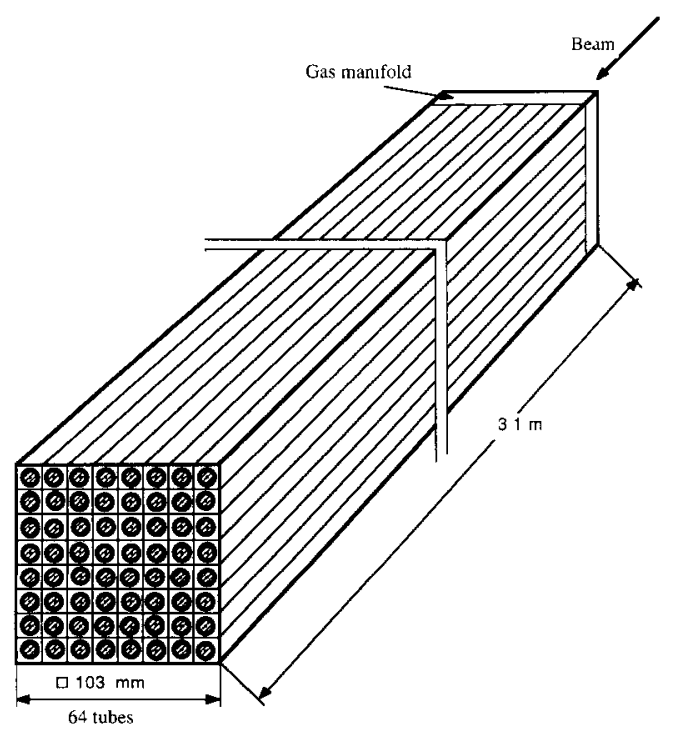

Fig. 2. Perspective view of one calorimeter module.

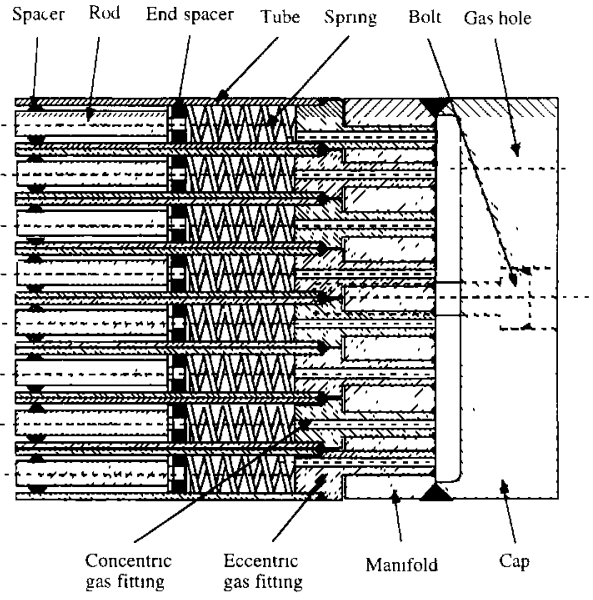

Fig. 3. Longitudinal cross-section of the front end of a module.

tubes are made eccentric to avoid dead space between neighbouring modules.

The module is assembled in the following sequence. First, each tube is assembled, welded, and tested for gas leaks and electrical breakdown. Then the gas-fitting ends of the tubes are inserted into the holes of the manifold and welded on the internal side of the manifold (Fig. 3). After another leak test the manifold is closed with a $25.4 \mathrm{~mm}$ thick cap and welded peripherally. For extra safety the cap is bolted to the manifold with five $8 \mathrm{~mm}$ diameter bolts which are welded to the cap's outside surface for gas sealing. The spaces between the tubes are filled with square steel rods as shown in Fig. 4.

Fourteen modules were stacked in the test calorimeter as shown in Fig. 4. The average density of the calorimeter is $5.4 \mathrm{~g} / \mathrm{cm}^{3}$, the nuclear interaction length is $24.4 \mathrm{~cm}$, and the radiation length is $2.6 \mathrm{~cm}$. The volume ratio of the steel to the readout gas is 2.9 . The average amount of steel in the cap, the manifold, and tubes in front of the active

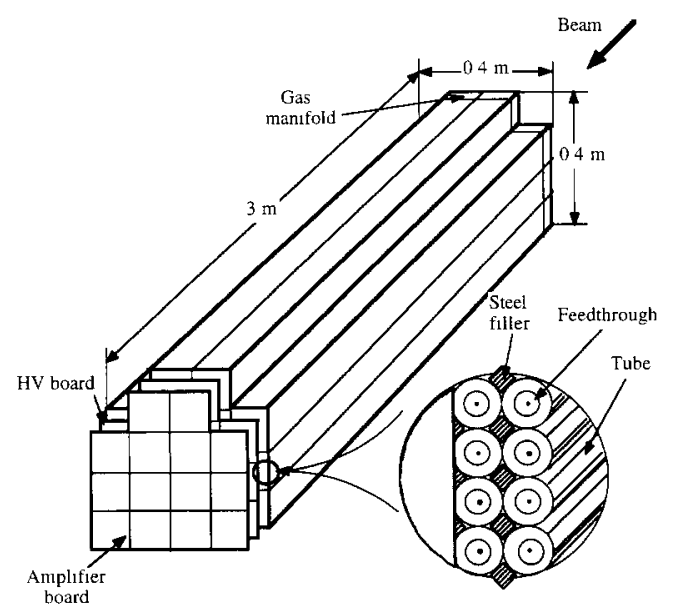

Fig. 4. Perspective view of the tested calorimeter. 
part of a module is $4.0 X_{0}$. The calorimeter was filled with a gas mixture of $95 \% \mathrm{Ar}+5 \% \mathrm{CH}_{4}$ at a pressure of 100 atm. The elements which are most sensitive to high pressure, the feedthrough and the manifold-cap unit, were tested hydraulically with pressures up to $640 \mathrm{~atm}$ without failure. The assembled calorimeter successfully passed a 167 atm gas pressure test at Fermilab and a similar test at CERN, in accordance with safety regulations.

\section{Test-beam setup}

The calorimeter was tested at the CERN-SPS X7B beam line in the West Hall in July 1993. We used negative pion and electron beams in the energy range of 10 to 100 $\mathrm{GeV}$. A scintillator telescope defined a $5 \times 5 \mathrm{~mm}^{2}$ beam at the calorimeter front surface. The calorimeter was installed on a table parallel to the beam. During the measurements we varied the horizontal angle between the calorimeter axis and the beam from $0.9^{\circ}$ to $9.1^{\circ}$, but the angle in the vertical plane was kept close to $0^{\circ}$. At each angle the horizontal position of the calorimeter was adjusted so that the hadron shower maximum (at about $1 \mathrm{~m}$ calorimeter depth) was approximately at the center of the calorimeter cross-section. The accuracy of the angle determination was $\pm 0.05^{\circ}$, and that of the calorimeter positioning was \pm 2 $\mathrm{mm}$.

\section{Front-end electronics and calibration}

A diagram of the amplifier circuit is shown in Fig. 5. Each input transistor was connected to two calorimeter tubes through a $4.7 \mathrm{nF}, 3 \mathrm{kV}$ capacitor. The circuit has a $12 \Omega$ impedance to match the $24 \Omega$ tube impedance. A resistor-diode chain was inserted between the capacitor and the amplifier input to protect the amplifier from accidental sparks. The circuit shown in Fig. 5 collects signals from an array of $4 \times 4$ tubes. Signals from four amplifiers connected to one module were summed in an

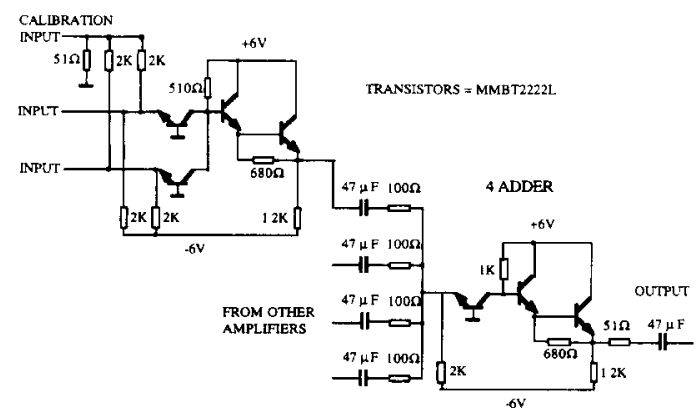

Fig. 5. Circuit design of the amplifiers and the four-fold adder. external adder. No signal shaping was done, and the ADC measured the signal charge integrated over the gate time, typically $80 \mathrm{~ns}$ (see below).

The rise time of the amplifier was about $15 \mathrm{~ns}$. The electronic noise from one module, integrated over the 80 ns gate time, was equivalent to about $16 \mathrm{fC}$ (r.m.s.) signal when amplifiers were connected to the module, and about $10 \mathrm{fC}$ when amplifiers were disconnected. A signal amplitude of $16 \mathrm{fC}$ amplitude corresponds to about $1 \mathrm{GeV}$ in energy for a hadron shower, or $0.8 \mathrm{GeV}$ for an EM shower (see Section 5, below). Thus, the expected noise for the whole calorimeter is $1 \mathrm{GeV} \times \sqrt{14}=3.7 \mathrm{GeV}$. However, in the test-beam environment, with long cables connecting the amplifiers to the $\mathrm{ADC}$, a coherent noise was picked up, and the total noise for the whole calorimeter was $7.9 \mathrm{GeV}$. The electronic noise for 6 modules, which contains $80 \%$ of the hadron shower, was $4.0 \mathrm{GeV}$. This should be compared with the expected sum of the amplifier noise of $2.5 \mathrm{GeV}$ for 6 modules.

During the data taking the electronic system was periodically calibrated with a $31 \mathrm{~ns}$ width rectangular signal of known amplitude distributed between all the amplifier inputs. Triggers without signal at the inputs were used to determine pedestals. An analysis of the calibration measurements showed that after a half-hour warm-up the gains were stable within $0.5 \%$ accuracy. However, the pedestal positions of individual modules varied by up to one ADC count (corresponding to about $0.25 \mathrm{GeV}$ of shower energy) between calibration runs. This uncertainty in the pedestal position could result in a considerable error in energy, especially in hadron measurements where the signals from all the 14 modules were summed. Fortunately, the pedestal drifts of different modules were synchronous, and during the entire run the differences between the pedestal positions of different modules were constant to within \pm 0.2 counts. We exploited this correlation by using one of the peripheral calorimeter modules to determine the pedestal position for the other modules during the beam runs. In the case of the electron beam, the particle shower was narrow and did not reach the peripheral modules, thus their signal amplitudes correspond to the true pedestal positions. In the case of the pion beam we used muon events (which contaminate the pion beam) for pedestal determination in the peripheral modules. The resulting error in the shower energy determination because of the pedestal uncertainty is $\pm 0.2 \mathrm{GeV}$.

In the process of data analysis the pedestals were subtracted from the corresponding channel pulse-heights. The results were then multiplied by individual channel calibration factors which convert ADC counts into charge. Finally, the amplitudes were corrected for the pressure drop $(0.2 \%$ per hour, due to a small leak in the outer gas system) on an event-by-event basis. The actual number of modules whose signal amplitudes were summed to form the calorimeter response depended on incident particle type, beam energy and tilt angle. 


\section{Calorimeter response to pions and electrons}

An oscilloscope trace of the amplifier output signal for $50 \mathrm{GeV}$ electrons for a calorimeter voltage of $1.2 \mathrm{kV}$ is shown in Fig. 6. The signal's full width at this voltage is about $80 \mathrm{~ns}$, and the width of the ADC gate signal (also shown in the figure) was adjusted accordingly. The dependence of the signal full width on the voltage is shown in Fig. 7, together with the calculated electron collection time for the $1.6 \mathrm{~mm}$ gas gap. The calculations are based on our published electron drift velocity measurements [5]. The working voltage of $1.2 \mathrm{kV}$ was chosen as the point where the collection time reaches its minimum, of about $38 \mathrm{~ns}$. The output signal is wider because of the amplifier response characteristics, and because of an approximately 10 ns difference in propagation times of the direct and reflected waves along the tube.

The dependence of the signal amplitude on the calorimeter voltage for $50 \mathrm{GeV}$ electrons is shown in Fig. 8 . These measurements were made with a variable gate width set equal to the signal width at each voltage. The signal amplitude saturates at a low voltage of about $300 \mathrm{~V}$. However, there is a signal drop at higher voltages, which may be explained by electron attachment to some impurities in the gas. There is an indication [12] that for some electronegative molecules the electron attachment crosssection increases with the electron energy. The pressure dependence of the calorimeter response to $50 \mathrm{GeV}$ electrons (Fig. 9) is also consistent with the presence of electronegative impurities (or recombination) in our calorimeter.

The calorimeter response to electrons and pions as a function of the beam energy is shown in Fig. 10. The data were taken with a calorimeter tilt angle of $9.1^{\circ}$. Very similar results were obtained at tilt angles of $0.9^{\circ}$ and $2.9^{\circ}$. The average signal amplitudes presented in these plots

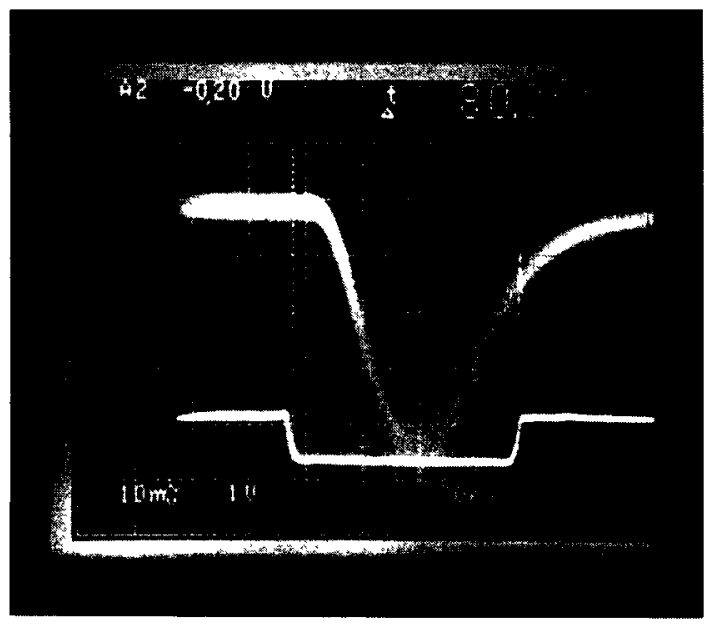

Fig. 6. Oscilloscope trace of the calorimeter output signal for 50 $\mathrm{GeV}$ electrons. The lower trace shows the gate signal.

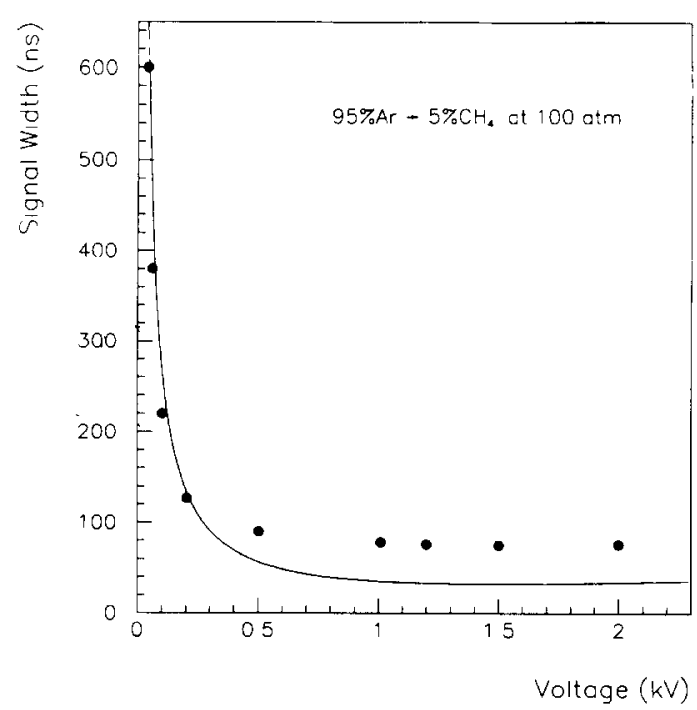

Fig. 7. Signal width at the base as a function of voltage for 50 $\mathrm{GeV}$ electrons (black dots). The line shows the calculated electron collect:on time dependence on voltage for a $1.6 \mathrm{~mm}$ gas gap [5].

were corrected for average energy losses in the calorimeter's front plates (gas manifold plus an extra $6 \mathrm{~mm}$ lead plate) for the electron data, and for lateral shower leakage for the pion data. The corrections were calculated by Monte Carlo simulations of the calorimeter. The EM energy losses for the calorimeter tilt angle of $9.1^{\circ}$ are tabulated in Table 1 . The calculated lateral hadron energy leakage shows little dependence on the pion beam energy. The leakage was about $7 \%$ for the tilt angles of $0.9^{\circ}$ and $2.9^{\circ}$, and about $11 \%$ for $9.1^{\circ}$.

The electron data presented in Fig. 10 demonstrate a very good linearity. The average charge per deposited energy of $19.6 \pm 0.1 \mathrm{fC} / \mathrm{GeV}$ can be compared with the calculated value of $25.1 \mathrm{fC} / \mathrm{GeV}$. The calculation is based

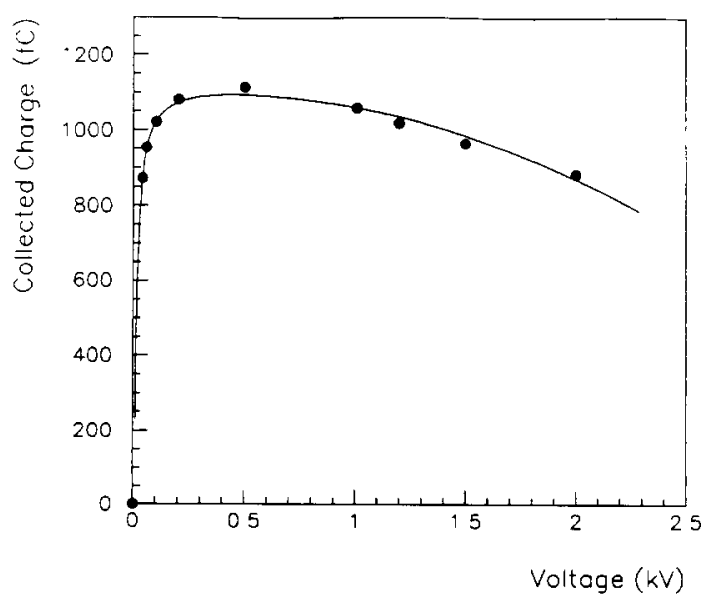

Fig. 8. Collected charge as a function of voltage, for $50 \mathrm{GeV}$ electrons. The pressure was $100 \mathrm{~atm}$. 


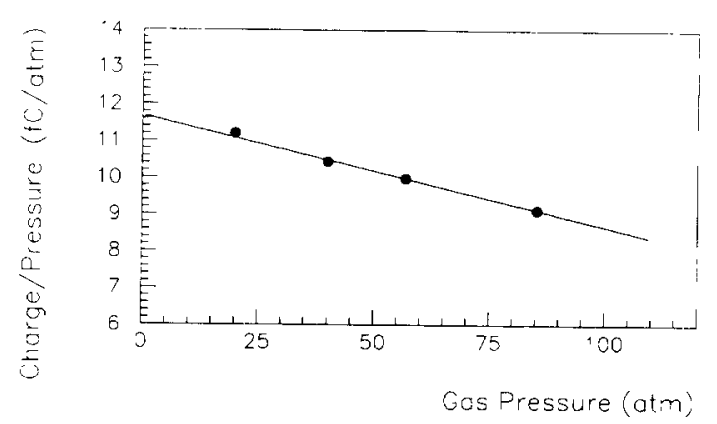

Fig. 9. Collected charge normalized to gas pressure as a function of pressure. The working voltage was proportional to the pressure at each point, with the voltage-to-pressure ratio equal to 12 $\mathrm{V} / \mathrm{atm}$.

on the mass ratio of active and passive materials $(0.745 \times$ $10^{-2}$ ), the ratio of ionization losses in $\mathrm{Ar}$ and $\mathrm{Fe}(1.05)$, the e/mip ratio (0.92) [13], the average ionization energy of $\operatorname{Ar}(26 \mathrm{eV})$, and the ratio of the charge induced on the electrodes during the electron collection time to the total charge of electrons in the gap, which is 0.567 for our tube design. The difference between the calculated and experimental values is believed to be due to electron attachment.

The pion data show a small nonlinearity due to the well-known decrease of the $e / h$ ratio with increasing energy [13]. This is illustrated in Fig. 11 where values of the $e / h$ ratio taken from Fig. 10 are plotted together with our Monte Carlo results.

\section{Hadron energy resolution}

Fig. 12 shows the pulse-height distribution for the 75 $\mathrm{GeV}$ pion beam. The spectrum contains two peaks corresponding to pions and muons. At energies of $50 \mathrm{GeV}$ and

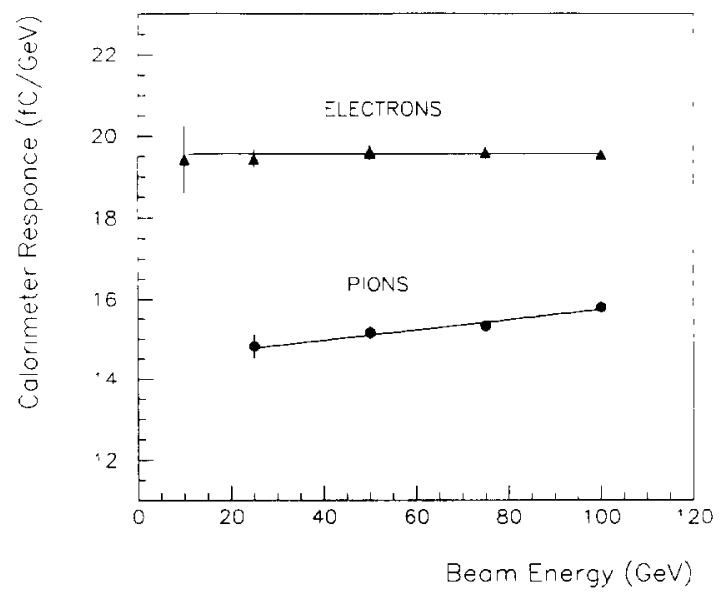

Fig. 10. Collected charge over beam energy for electrons (triangles) and pions (circles), as a function of beam energy.
Table 1

EM energy loss in the front plates of the calorimeter $\left(5.1 X_{0}\right)$ (Monte Carlo)

\begin{tabular}{llllll}
\hline Electron & 10 & 25 & 50 & 75 & 100 \\
Energy loss (\%) & 33.9 & 24.9 & 19.6 & 16.8 & 14.9 \\
\hline
\end{tabular}

above, the peaks are well separated and the muon contamination is not a problem. However, we were forced to discard the measurement at $10 \mathrm{GeV}$ because the two peaks merged. The experimental errors at $25 \mathrm{GeV}$ were also high due to the muon-pion separation uncertainty.

The width of the pion peak is the result of intrinsic signal fluctuations, electronic noise, and lateral leakage fluctuations. To obtain the intrinsic calorimeter resolution we quadratically subtracted the electronic noise width and corrected the results for the lateral shower leakage using Monte Carlo simulations. The final results are shown in Fig. 13(a). Data for three calorimeter tilt angles $\left(0.9^{\circ}, 2.9^{\circ}\right.$ and $9.1^{\circ}$ ) have been combined because the Monte Carlo simulations (Table 2) predict a negligible dependence of the pion energy resolution on the angle. Furthermore, the experimental errors, especially at low energies, are too large to allow a meaningful fitting procedure for the individual angle data. The result of fitting the combined experimental pion data is

$\delta E / E=(70 \pm 12) \% / \sqrt{E} \oplus(7.4 \pm 1.2) \%$.

The constant term is caused by the inequality of the calorimeter response to electrons and hadrons. The constant term is slightly higher for pions than for protons because charged pions produced more neutral pions (which convert into EM energy) in the first interaction.

A software method of $e / h$ compensation for a wellsegmented calorimeter was developed by the $\mathrm{H} 1$ Calorimeter Group (see Ref. [14] and references therein). Although our calorimeter has only transverse segmentation, the following analysis demonstrates that even this type of segmentation could be helpful. We proceed from the idea that most of the EM showers, or at least the most energetic ones, are concentrated along the core of the hadron shower.

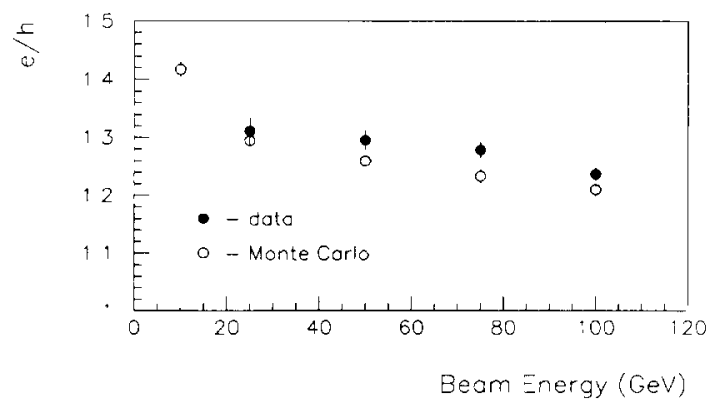

Fig. 11. The $e / h$ ratio as a function of energy: experimental results (black points) and Monte Carlo simulations (open circles). 


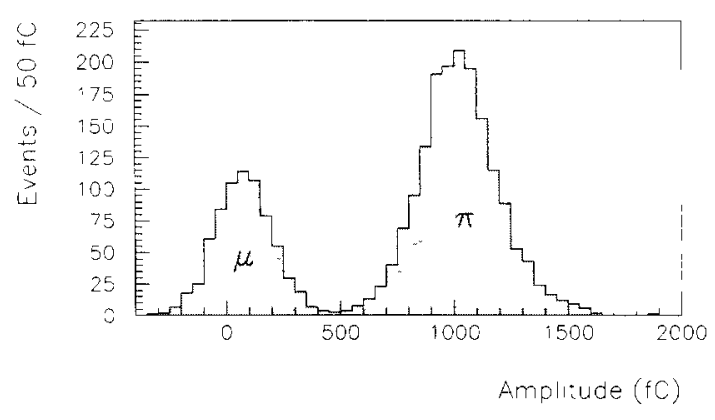

Fig. 12. Pulse-height spectrum for a $75 \mathrm{GeV}$ pion beam at a tilt angle of $0.9^{\circ}$.

Hence, one can expect a decrease in the signal fluctuations by suppressing the signals from that part of the shower. In Fig. 14 the calorimeter energy resolution for $100 \mathrm{GeV}$ pions is plotted versus a suppression coefficient, $k$, which was applied to the two central modules containing about $70 \%$ of the shower energy. The dependence shows a clear minimum at $k=0.6$. This coefficient was applied to all the data presented in Fig. 13(a), independent of the beam energy and tilt angle, though the optimum value of $k$ is expected to depend slightly on both of these parameters. The results are shown in Fig. 13(b). The best fit in this

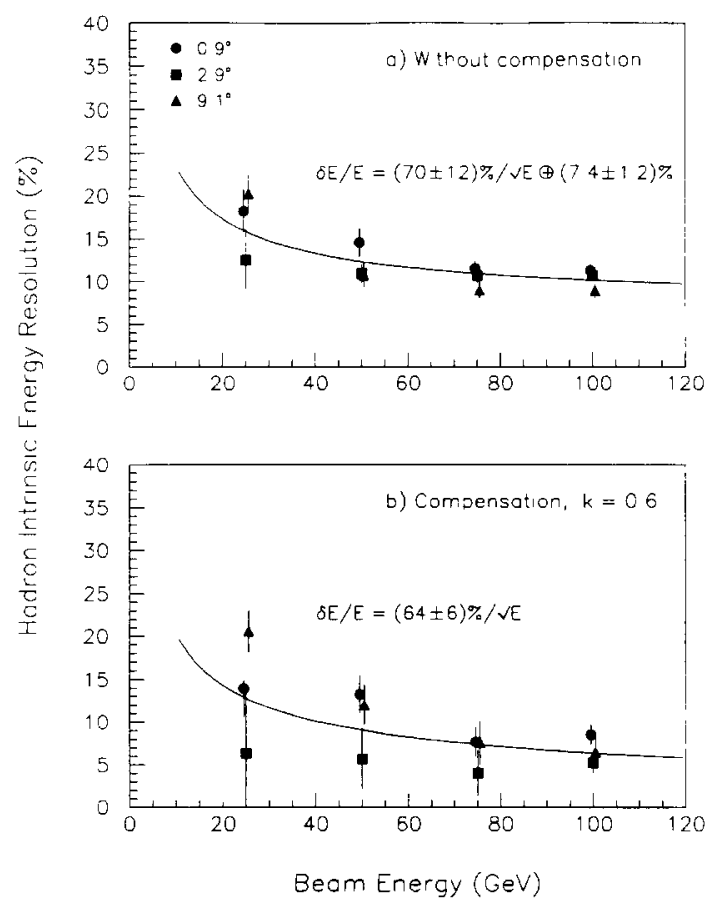

Fig. 13.(a) Calorimeter energy resolution for pions, as a function of beam energy. Data are corrected for electronic noise and lateral shower leakage fluctuations. The solid curve is the result of a fit to all points, independently of tilt angle. (b) The same, but after a compensation procedure was applied (see text).

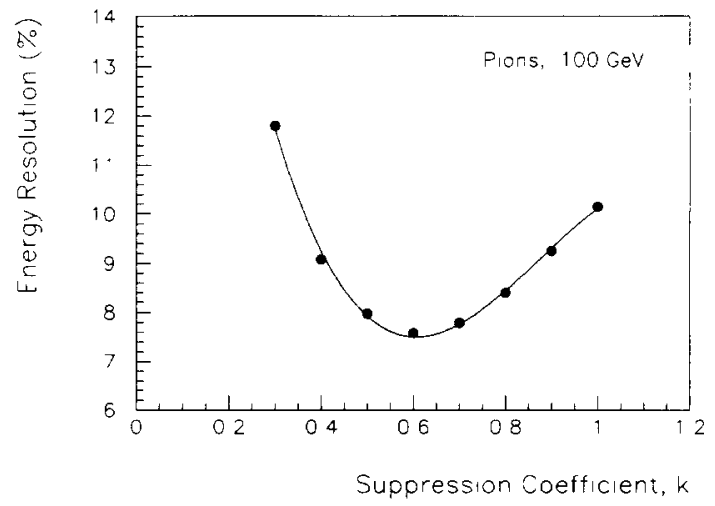

Fig. 14. Pion energy resolution at $100 \mathrm{GeV}$ as a function of the suppression coefficient $k$ applied to the two central modules. Data are averaged over tilt angle and corrected for electronic noise and lateral shower leakage.

case does not include any constant term. However, we cannot exclude a constant term of $\leq 3 \%$. The final conclusion is that with the software compensation the calorimeter's intrinsic hadron resolution is

$\delta E / E=(64 \pm 6) \% / \sqrt{E}$,

with a possible constant term of $\leq 3 \%$.

\section{Electromagnetic energy resolution}

The relatively coarse structure of this tube design, which was optimized for hadrons, causes increased signal fluctuations for the comparatively narrow EM showers. The pulse-height spectra and resolution strongly depend on the position and angle of the incident electron. Two pulseheight spectra for $75 \mathrm{GeV}$ electron beam at angles $9.1^{\circ}$ and $0.9^{\circ}$ are shown in Fig. 15. One can see a double-peaked distribution in the $0.9^{\circ}$ spectrum which is a result of different calorimeter responses to beam electrons hitting different points of the calorimeter front surface. The signals from just four modules were summed for these spectra and for the following analysis, because of the narrowness of the EM shower. To determine the intrinsic EM resolution we calculated the r.m.s. of the pulse-height spectrum, quadratically subtracted the electronic noise, and corrected the result for the energy loss in the calorimeter front part, as follows.

The uncorrected calorimeter response to electrons is not linear with the beam energy because of this loss (see Table 1). According to our Monte Carlo simulations the energy dependence of the signal amplitude, $A(E)$, can be described by the empirical formula

$A(E)=a E\left(1-b E^{-c}\right)$,

where parameters $b$ and $c$ depend on the thickness of the absorber material in front of the calorimeter, and are 
Table 2

Monte Carlo results for the calorimeter hadron resolution

\begin{tabular}{lcccc}
\hline & Pions & & \multicolumn{2}{c}{ Protons } \\
\hline Tilt angle & 0.9 & 2.9 & 9.1 & 2.9 \\
Stochastic term, $(\% \times \sqrt{E})$ & $66.5 \pm 2.2$ & $65.4 \pm 2.7$ & $67.5 \pm 2.6$ & $74.2 \pm 1.4$ \\
Constant term $(\%)$ & $8.30 \pm 0.35$ & $6.85 \pm 0.47$ & $6.94 \pm 0.44$ & $5.14 \pm 0.31$ \\
\hline
\end{tabular}
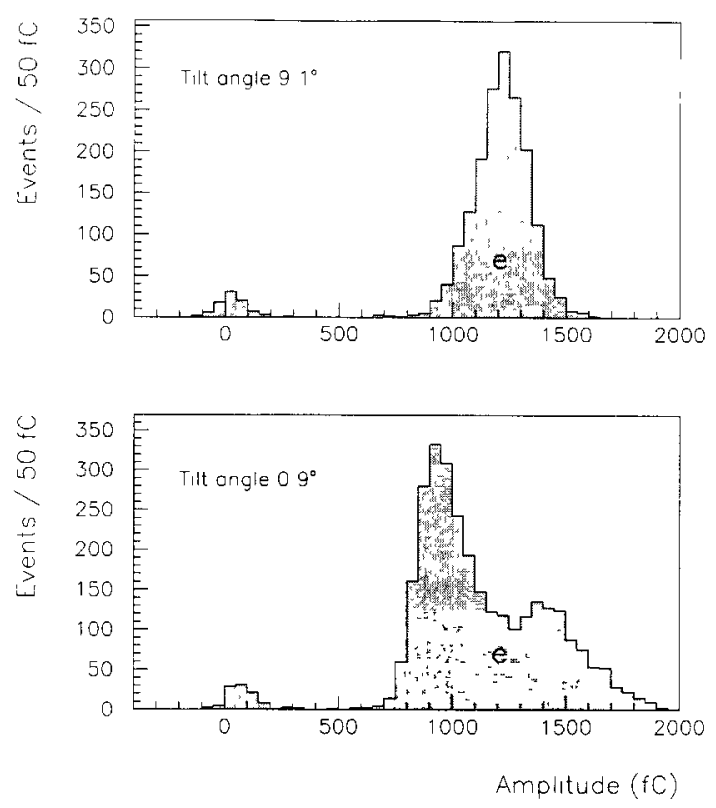

Fig. 15. Pulse-height distribution for $75 \mathrm{GeV}$ electrons at tilt angles of $9.1^{\circ}$ and $0.9^{\circ}$.

tabulated in Table 3. The energy resolution $(\delta E / E)$ is related to the relative width $(\delta A / A)$ of the signal distribution by the relation

$$
\frac{\delta E}{E}=\frac{A(E)}{E \frac{\mathrm{d} A}{\mathrm{~d} E}} \frac{\delta A}{A} \equiv G(E) \frac{\delta A}{A},
$$

where $G(E)$ is the correction factor.

The intrinsic EM energy resolution as a function of energy, for three values of tilt angle, is shown in Fig. 16. The data were fitted with the expression:

$\delta E / E=a / \sqrt{E} \oplus b$.

The parameters for each angle are presented in Fig. 16. One can see a strong dependence of the constant term on the tilt angle, though the stochastic term is relatively insensitive to this parameter. We compare these results with the Monte Carlo calculations made with a "wide beam" $\left(4 \times 4 \mathrm{~cm}^{2}\right)$ which averages the calorimeter response over the position of the incident electrons. The calculated resolution depends not only on the incident
Table 3

Coefficients for formula 3 (energy in $\mathrm{GeV}$ ), and the correction factor $G(E)$ for energies $E=10 \mathrm{GeV}$ and $E=100 \mathrm{GeV}$

\begin{tabular}{lllll}
\hline Absorber & $b$ & $c$ & $G(10)$ & $G(100)$ \\
\hline Calorimeter manifold, $4 X_{0}$ & 0.53 & 0.44 & 0.906 & 0.964 \\
Plus 6 mm lead, 5.1 $X_{0}$ & 0.71 & 0.33 & 0.856 & 0.936 \\
Plus 12 mm lead, 6.1 $X_{0}$ & 0.89 & 0.29 & 0.806 & 0.910 \\
Plus 18 mm lead, 7.2 $X_{0}$ & 0.98 & 0.22 & 0.764 & 0.883 \\
Plus 24 mm lead, 8.3 $X_{0}$ & 0.99 & 0.17 & 0.736 & 0.862 \\
\hline
\end{tabular}

particle polar angle but also on its azimuthal angle $(\phi)$ as demonstrated in Fig. 17. For this reason we present in Fig. 18 the calculated stochastic and constant terms for the EM energy resolution versus the polar tilt angle for two values of the azimuth, $\phi=0$ and $\phi=\pi / 4$, which outline ranges of possible resolution parameters. The black points in this figure are the experimental results (from Fig. 16) obtained with a "narrow beam" $\left(5 \times 5 \mathrm{~mm}^{2}\right)$.

In Ref. [6] a method to reduce the EM constant term of the tube calorimeter was proposed. The idea is to spread the energy of the EM particle over the face of the calorimeter by means of a preshower converter placed in front of the calorimeter. In Fig. 19 experimental data on the calorimeter energy resolution for $100 \mathrm{GeV}$ electrons at the tilt angle $0.9^{\circ}$ are plotted as a function of the preshower thickness. The distance between the preshower and the calorimeter was $78 \mathrm{~cm}$. The expected improvement was not obtained because of the $4 X_{0}$ of material in the front part of the calorimeter which cannot be moved to the proper distance from the active region of the calorimeter.

The pressure dependence of the calorimeter energy resolution for $50 \mathrm{GeV}$ electrons at $7.3^{\circ}$ tilt angle is shown in Fig. 20 together with the Monte Carlo calculations. No significant pressure dependence is observed.

\section{Muon identification}

The nonuniform transverse structure of this calorimeter and comparatively large electronic noise does not favor muon identification. However, it is not impossible, as demonstrated in Fig. 21 which shows a muon pulse-height spectrum from two modules at a tilt angle of $2.9^{\circ}$, along with the superimposed pedestal. The overlap of the two 


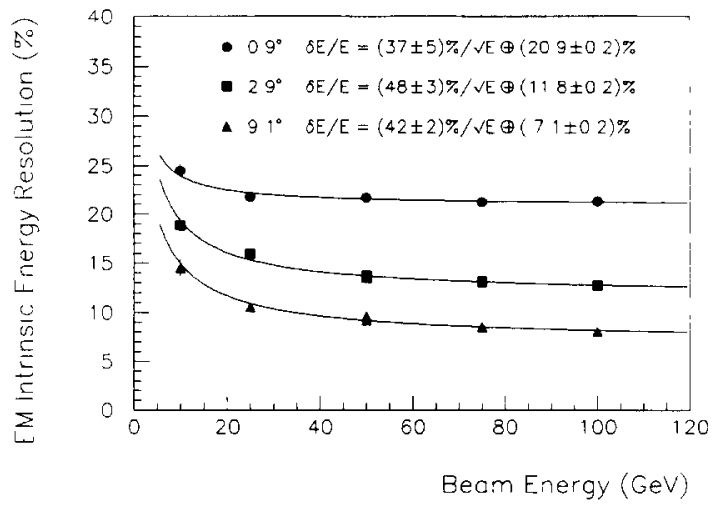

Fig. 16. Energy resolution for electrons as a function of beam energy, for various calorimeter tilt angles. Data are corrected for electronic noise and energy loss in the calorimeter front plates. The solid curves represent fits to the data points.

peaks is about $10 \%$, which could be reduced by decreasing the number of tubes connected to one electronic channel.

\section{Summary}

The main result of this test is that this new type of calorimeter proved to be safe and easy to operate, and worked smoothly during the entire beam test. The calorimeter response is linear with energy. The electron collection time for a $95 \% \mathrm{Ar}+5 \% \mathrm{CH}_{4}$ gas mixture is about 38 ns [5]. The full signal width without shaping was 80 ns in this test, but with proper signal shaping we anticipate a $25 \mathrm{~ns}$ signal with little degradation in the signal-to-noise ratio. The intrinsic hadron energy resolution compares well with calorimeters based on scintillator or liquid argon readout. We expect a resolution of about

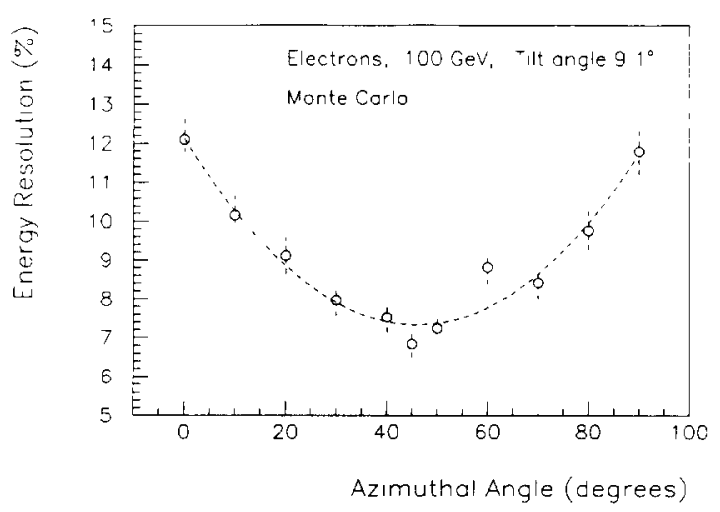

Fig. 17. Monte Carlo calculations of the EM resolution dependence on azimuthal angle of the incident particle.
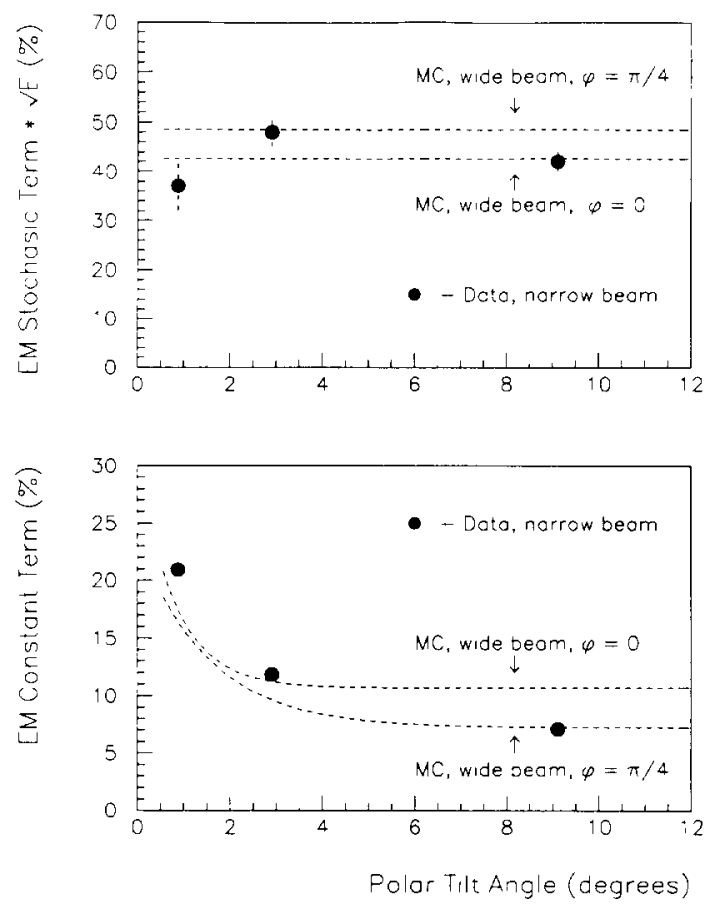

Fig. 18. Comparison of the experimental results on EM resolution (black points) with Monte Carlo calculations (dashed lines). The definitions of narrow and wide beams are discussed in the text.

$3-4 \%$ at energies above $500 \mathrm{GeV}$, which are typical in the LHC forward region. The measured electronic noise is negligible at these energies. The EM resolution is not as good because the calorimeter design was optimized for hadrons, not for electrons. At present we are designing a high-pressure calorimeter which will have better EM reso-

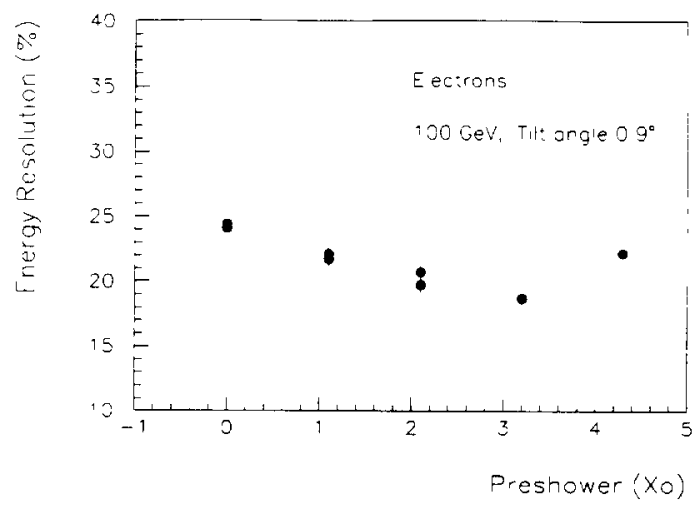

Fig. 19. EM energy resolution as a function of the preshower converter thickness. The preshower was placed $78 \mathrm{~cm}$ in front of the calorimeter. 


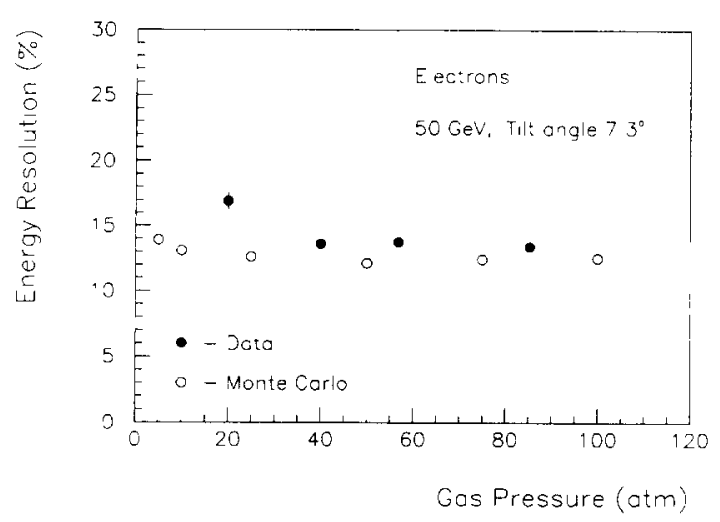

Fig. 20. EM energy resolution as a function of pressure: experimental results (black points) and Monte Carlo calculations (open circles).

lution. The main differences of this new design are: a smaller tube diameter, a hexagonal arrangement of the tubes, a wiggling tube profile, and a reduced thickness of the gas manifold.

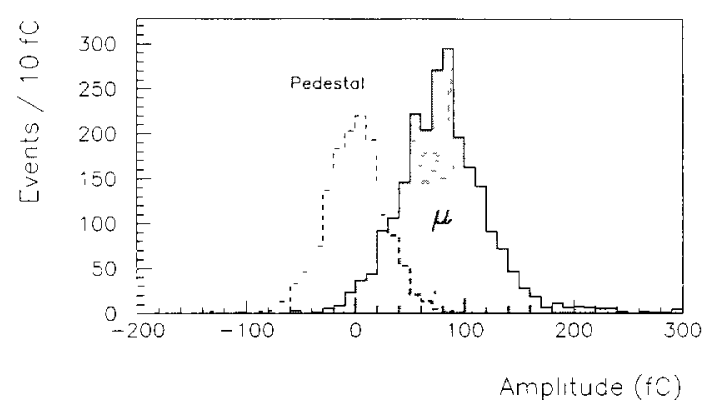

Fig. 21. Muon pulse-height spectrum from two modules. The corresponding noise distribution (pedestal) is shown in dashed line.

\section{Acknowledgements}

This work was supported by the U.S. Department of Energy, the National Science Foundation, and the Texas National Research Laboratory Commission. We thank the Fermilab Physics Department for providing support during the construction of the calorimeter. We are grateful to Drs. V. Radeka, S. Hansen and C. Riveta for very productive discussions on the calorimeter electronics design and for their practical help. We are thankful to I. Stauersboll whose excellent welding job provided for smooth construction and testing of the calorimeter. We also thank the CERN beam support personnel, especially R. Dillon and L. Gatignon, for their help during the test.

\section{References}

[1] N.D. Giokaris et al., Nucl. Instr. and Meth. A 291 (1990) 552.

[2] D.M. Khazins et al., Nucl. Instr. and Meth. A. 300 (1991) 281.

[3] L. Demortier et al., Nucl. Instr. and Meth. A 324 (1993) 77.

[4] D.M. Khazins, Nucl. Instr. and Meth. A 330 (1993) 316.

[5] N.D. Giokaris et al., Nucl. Instr. and Meth. A 333 (1993) 364.

[6] D M. Khazins et al., Nucl. Instr. and Meth. A 333 (1993) 372.

[7] L. Demortier et al., to be submitted to Nucl. Instr. and Meth.

[8] S. Cihangir et al., IEEE Trans. Nucl. Sci. NS-36(1) (1989) 347.

[9] V.I. Baskakov et al., Nucl. Instr. and Meth. 159 (1979) 83.

[10] S. Denisov et al., Nucl. Instr. and Meth. A 335 (1993) 106.

[11] V.E. Konstantinov et al., ATLAS Internal Note CAL-NO-027 (1993).

[12] R.A. Holroyd, The Electron: Properties and Reactions, in: Radiation Chemistry, eds. Farhataziz and M.A.J. Rodgers (VCH, Dearfield Beach, FL, 1987).

[13] R. Wigmans, Nucl. Instr. and Meth. A 259 (1987) 389

[14] H1 Calorimeter Group, Nucl. Instr. and Meth. A 336 (1993) 499. 